\section{Orbital eccentricity in classical novae}

\section{A. Edwards \& J. E. Pringle*}

Lick Observatory, Board of Studies in Astronomy and Astrophysics, University of California, Santa Cruz, California 95064, USA

* Permanent address: Institute of Astronomy, Madingley Road, Cambridge CB3 OHA, UK

The classical nova outburst is caused by a thermonuclear explosion on the surface of a white dwarf ${ }^{1,2}$. The explosion ignites at the base of an envelope of hydrogen-rich material which has been accreted from a binary companion star. It has long been evident, however, that in the explosion most of the envelope is not consumed by nuclear burning, but is ejected from the system. This is because to consume the envelope mass $\left(\sim 10^{-4} M_{\odot}\right)$ at the maximum possible rate (with luminosity equal to the Eddington limit) would take $\sim 300$ years, whereas classical novae return to their preoutburst brightness within $\sim 10$ years (ref. 3 ). Rough confirmation of this is given by the fact that the masses of shells of material observed to be ejected from novae are in the range $10^{-3}$ to $10^{-5} \mathrm{M}_{\odot}$ (ref. 4). Here we consider the effect on the orbital parameters of a classical nova of the ejection of mass during the nova explosion. The most easily observable consequence is the generation of a small eccentricity in the orbit which leads to a luminosity modulation at a period just longer than the orbital period. Observation of such an effect, would have implications not just for interpreting the dynamics of the explosion but also for measuring the secular effect of tidal interaction after the outburst.

There has been considerable speculation as to how this ejection takes place. The initial force of the explosion is capable of ejecting at least some material in a dynamical fashion, and numerical hydrodynamical calculations have demonstrated that up to about a half of the envelope mass can be ejected in this way $^{5}$. The ejection of the remainder of the envelope appears to take place on a longer timescale in the form of a substantial wind, driven either by radiation pressure ${ }^{6}$ or by interaction of the extended atmosphere of this white dwarf with its binary companion $^{3,7}$. It seems likely that the fraction of the envelope which is ejected promptly depends on the speed class of the nova.

The rapid ejection of matter from the white dwarf has dynamical consequences for the orbit of the binary, which in turn leads to observational effects. For the sake of argument we consider a nova system to consist of a white dwarf of mass $M_{1}=1 M_{\odot}$, and a secondary star of mass $M_{2}=0.6 M_{\odot}$, effective surface temperature $T_{2}=4,500 \mathrm{~K}$ and equivalent volume radius $R_{2}=$ $3.7 \times 10^{10} \mathrm{~cm}$. We assume the secondary fills its Roche lobe, so that the binary separation is $a=1.1 \times 10^{11} \mathrm{~cm}$ and the period is $P=4.4 \mathrm{~h}$. Before and well after the nova explosion, the main optical light from the system is due to the accretion luminosity caused by a steady flow of mass from the secondary onto the white dwarf. If before outburst the mass transfer rate is $\boldsymbol{M}=$ $10^{-8} M_{\odot} \mathrm{yr}^{-1}$, then at that time the secondary overfills its Roche lobe by an amount $\Delta R=7.2 \times 10^{7} \mathrm{~cm}$ (refs 8,9$)$.

An ejection of mass from the binary affects the orbit in such a way as to produce two distinct observable consequences. First,

\section{Received 20 May; accepted 16 June 1987}

Gallagher, J. S. \& Starrfield, S. A. Rev. Astr. Astrophys. 16, 171-214 (1978).

2. Truran, J. W. in Essays in Nuclear Astrophysics (ed. Barnes, C.) 467-495 (Cambridge University Press, 1985)

3. MacDonald, J., Fugimoto, M. Y. \& Truran, J. W. Astrophys. J. 294, 263-270 (1985)

4. McLaughlin, D. B. in Stars and Stellar Systems Vol. VI, (ed. Greenstein, J. L.) 585-653 (University of Chicago Press, 1960).

5. Starrfield, S., Truran, J. W. \& Sparks, W. M. Astrophys. J. 226, 186-202 (1978)

6. Bath, G. T. \& Shaviv, G. Mon. Not. R. astr. Soc. 175, 305-322 (1976). if the mass is ejected promptly from the white dwarf, the binary separation increases ${ }^{10,11}$ by $\Delta a \sim a \Delta M /\left(M_{1}+M_{2}\right)$. For $\Delta M=$ $10^{-4} M_{\odot}$ and the above parameters this leads to a decrease in $\dot{M}$ by about $9 \%$. This is consistent with the observation ${ }^{12}$ that the pre- and post-eruption luminosities (due to accretion) are the same to within observational error. Moreover, if the ejection is not prompt but takes place slowly enough that the matter can interact tidally with the secondary star as it leaves the system ${ }^{3,7}$, then $\Delta a$ is less than the estimate given above, and may even be negative, leading to an increase in $\dot{M}$. There are also other effects, such as direct heating of the secondary by the hot white dwarf remnant ${ }^{11}$ which may affect the post outburst mass transfer rate. Thus a change in mass transfer rate post outburst even if observed is not readily interpretable.

Second, however, a prompt ejection of mass from the primary leads to an eccentricity $e$ in the binary orbit. If the timescale on which the mass $\Delta M$ is ejected is $t_{\mathrm{ej}}$, then the eccentricity induced is ${ }^{13}$

$$
e \simeq \frac{\Delta M}{M_{1}} \times \min \left\{1, P / 2 \pi t_{\mathrm{ej}}\right\}
$$

For the parameters given above, the maximum eccentricity generated by $\Delta M=10^{-4} M_{\odot}$ is $e \simeq 10^{-4}$. Even such a small orbital eccentricity has observable consequences ${ }^{9,14}$ as it leads to a regular variation in mass transfer rate, and hence of system brightness. For $e \simeq 10^{-4}$ the overall variation in $\dot{M}$ is $31 \%$, and is $1 \%$ even for $e \simeq 3 \times 10^{-6}$. We note that since the variation takes place on a period which is slightly longer than (by a few per cent) but not equal to the orbital period ${ }^{14}$, the effect of such an eccentricity should be distinguishable from the orbital variation. Most of the eccentricity is generated by the amount of mass ejected dynamically in the explosion, for which $t_{\mathrm{ej}} \ll P$, and little is generated by the ensuing slow mass loss. Thus observation of the effect of such an eccentricity gives a direct measurement of the mass ejected promptly. We should also note that there are processes acting which serve to decrease the eccentricity. In particular the interaction of the wind with the secondary star mentioned above $\mathrm{e}^{3,7}$, and simple tidal forces on the secondary ${ }^{10}$ both act in the direction of decreasing the eccentricity. The timescales of both processes are quite uncertain. The wind interaction (if it occurs) is likely to act on a timescale comparable to the ejection of the envelope as a whole. The usual tidal effect is not likely to be rapid. For such a small eccentricity even for a lobe filling star, the tides do not induce supersonic motions, and the slow convective turnover times in the secondary lead to long tidal timescales ${ }^{15}$. Thus, the observation of an eccentricity in the orbit is not only of importance in understanding the dynamics of the nova explosion, but observation of its secular decrease would give the first direct measurement of the timescale on which tides act in a binary star system.

This work was carried out under the auspices of a special NASA astrophysics theory program which supports a joint centre for star formation studies at NASA-Ames Research Center, UC Berkeley, and UC Santa Cruz. We gratefully acknowledge support from NATO (D.A.E.) and the Fulbright Commission (J.E.P.).

This is Lick Observatory Contribution No. 445.
7. MacDonald, J. Mon. Not. R. astr. Soc. 191, 933-949 (1980).

8. Paczynski, B. \& Sienkiewicz, R. Acta, astr, 22, 73-92 (1972).

9. Edwards, D. A. \& Pringle, J. E. Mon. Not, $R$ astr. Soc (in the press).

10. Pringle, J. E. \& Wade, R. A. (eds) Interacting Binary Stars (Cambridge University Press, 1985)

11. Shara, M. M., Livio, M., Moffatt, A. F. J. \& Orio, M. Astrophys. J. (in the press).

12. Robinson, E. L. Astr. J. 80, 515-524 (1975).

13. Savedoff, M. P. \& Vila, S. Astr. J. 69, 242-245 (1964).

14. Papaloizou, J. C. B. \& Pringle, J. E. Mon. Not. R. astr. Soc. 189, 293-297 (1979).

15. Campbell, C. G. \& Papaloizou, J. C. B. Mon. Not. R. astr. Soc. 204, 433-447 (1983) 